\title{
Définir un cadre méthodologique commun en cartographie participative
}

L'atelier collectif de Cabrousse en Casamance (Sénégal), de la théorie à la pratique

Defining a Common Methodological Framework in Participatory Mapping: The

Collective Workshop of Cabrousse in Casamance (Senegal), from Theory to

Practice

\section{Marie-Christine Cormier-Salem et Tidiane Sané}

\section{OpenEdition}

\section{Journals}

Édition électronique

URL : http://journals.openedition.org/ethnoecologie/2930

DOI : $10.4000 /$ ethnoecologie.2930

ISSN : 2267-2419

Éditeur

Laboratoire Eco-anthropologie et Ethnobiologie

Référence électronique

Marie-Christine Cormier-Salem et Tidiane Sané, «Définir un cadre méthodologique commun en cartographie participative », Revue d'ethnoécologie [En ligne], 11 | 2017, mis en ligne le 03 juillet 2017, consulté le 19 avril 2019. URL : http://journals.openedition.org/ethnoecologie/2930 ; DOI : 10.4000/ ethnoecologie.2930

Ce document a été généré automatiquement le 19 avril 2019

\section{c) (i) $९$}

Revue d'ethnoécologie est mis à disposition selon les termes de la licence Creative Commons Attribution - Pas d'Utilisation Commerciale - Pas de Modification 4.0 International. 


\section{Définir un cadre méthodologique commun en cartographie participative}

L'atelier collectif de Cabrousse en Casamance (Sénégal), de la théorie à la pratique

Defining a Common Methodological Framework in Participatory Mapping: The

Collective Workshop of Cabrousse in Casamance (Senegal), from Theory to

Practice

Marie-Christine Cormier-Salem et Tidiane Sané

\section{Introduction : problématique de départ, objectifs et contexte d'intervention}

1 Les démarches participatives en général, et celles de cartographie participative en particulier, connaissent depuis les années 1960, un engouement très fort et se sont diversifiées et multipliées avec l'apport de nouveaux outils, tels le numérique (Arnstein 1969, Chambers 1991 et 2006, Gueye \& Schoonmaker-Freudenberger 1991, Palsky 2010, Burini 2005, Lavigne Delville 2011, Liagre \& Nonjon 2012, Amelot 2013).

2 Palsky (2010) définit la cartographie participative comme un processus d'élaboration collective des représentations cartographiques par la base, c'est-à-dire par un ensemble de personnes n'appartenant pas aux milieux de la cartographie professionnelle ou académique ; c'est ce qu'il appelle « cartographie sans cartographes ». Pour Burini (2008), il s'agit d'un type particulier de cartographie qui, réalisée par les communautés locales pour représenter leur territoire, permet de faire émerger les savoirs traditionnels et certaines questions qui échappent aux enquêtes traditionnelles. On retiendra la définition simple et consensuelle de Habert (2014), à savoir qu'il s'agit de la cartographie d'un territoire produite par un groupe d'habitants encadré par un groupe d'experts. À travers ces quelques définitions et au-delà des réalités très diverses auxquelles elles renvoient, il 
se dégage un consensus sur l'objectif poursuivi par la cartographie participative, celle de faire participer les populations locales à la représentation de leurs territoires et de produire les connaissances utiles à leur implication dans les actions de développement qui portent sur leurs localités. Elle concerne donc, comme l'atteste Palsky (2010), la production d'une information géographique (ou spatiale) par une communauté d'individus, dans une logique de " bottom-up ", c'est-à-dire de la base vers le sommet.

Dans la continuité des réflexions conduites par des membres de l'UMR PALOC, l'école thématique de Cartographie Participative (CP) dans la Communauté Rurale (CR) de Diembéring en Casamance (cf. Figure 1) a eu pour objectif de mener une analyse critique et réflexive sur ces démarches. Il s'est agi de partager nos expériences pour élaborer un cadre commun, l'appliquer sur le terrain et en tirer des leçons. Nous présentons ici le cadre méthodologique adopté et son application sur le terrain, les principaux résultats en termes de connaissances et d'actions, et enfin discutons les apports et limites de ces démarches, en particulier dans nos pratiques de recherche.

Figure 1 : Communauté rurale de Diembéring, Casamance, Sénégal

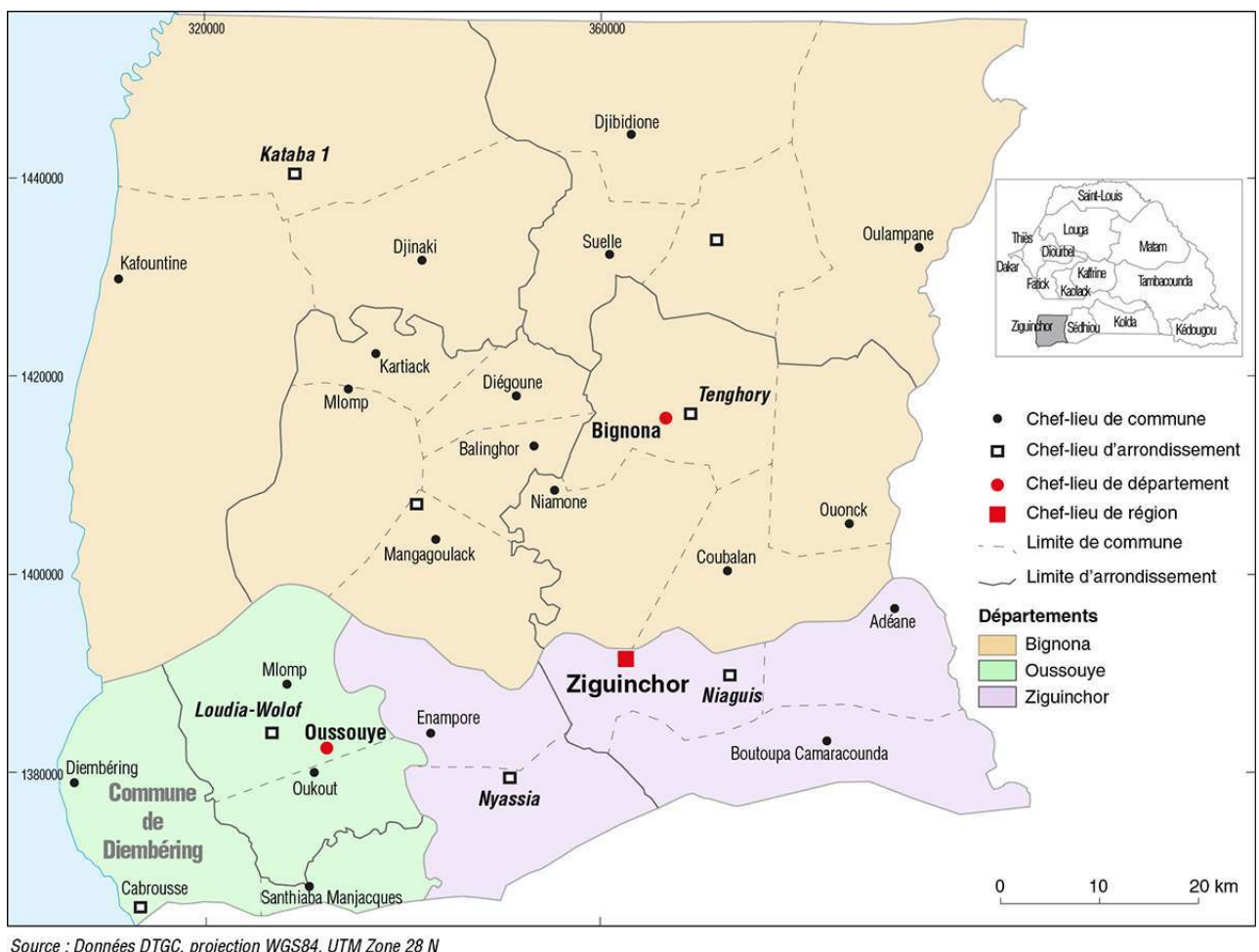

ce : Données DTGC, projection WGS84, UTM Zone 28

() T. Sané

\section{Pour un cadre méthodologique commun}

Du 30 juin au 4 juillet 2014, une école thématique de cartographie participative a été organisée en Casamance, au Sénégal, conjointement par l'UMR 208 PALOC, IRD/MNHN et le LMI PATEO, Sénégal ${ }^{1}$. L'école thématique a réuni un panel très diversifié de 30 participants, diversifiés de par leurs disciplines, institutions, implications, origines «locaux », nationaux et étrangers. Il s'agissait à la fois "d'experts » (chercheurs et enseignants-chercheurs, techniciens tels les géomaticiens et cartographes, étudiants) ${ }^{2}$, 
mais aussi de membres d'ONG, de représentants de la société civile, de gestionnaires et de producteurs de données spatiales, à savoir en priorité les acteurs locaux, dont les points focaux de la Communauté Rurale de Diembéring en Casamance.

L'action s'est déroulée en trois temps :

Un premier temps de deux jours de travaux en salle, théoriques et méthodologiques, a permis d'aborder les concepts et méthodes, échanger nos expériences, élaborer un cadre commun. Sept exposés ont été présentés, dont un général (E. Habert) et six sur des expériences de cartographie participative dans des contextes diversifiés : îles Marquises (F. Chlous), Amazonie brésilienne (P. de Robert), Timor oriental (D. Guillaud), Tanzanie et Kenya (S. Duvail), Mauritanie (Boubacar Ba El Abass), Sénégal (M. Fabre, L. Touré et M.C. Cormier-Salem), dont plusieurs ont fait l'objet de publication dans le numéro 9 de la Revue d'Ethnoécologie paru en avril 2016 et dans ce numéro. Le canevas, proposé au préalable aux intervenants pour faciliter les échanges, reposait sur les questions suivantes :

- Pour qui et avec quels acteurs? Genèse des projets

- Pour quoi faire? En réponse à quelle demande? Objectifs ? Définis par qui ?

- Comment ? Démarche mise en œuvre ; rôle respectif des acteurs ; outils mobilisés

- Principaux résultats : apports ; limites

- Produits et prospectives : suites données à ce projet.

Un second temps de deux jours de travaux sur le terrain, empiriques, a permis de tester le cadre commun sur une des zones ateliers de la convention programme de l'Agence Française de Développement/AFD "Gouvernance citoyenne des littoraux sud", coordonnée par le GRDR et impliquant les partenaires du LMI PATEO (IRD et UASZ). Trois villages de la Communauté Rurale ( $\mathrm{CR}$ ) de Diembéring (Diembéring, Bouyouye et Cabrousse) et deux thèmes (riz et riziculture, patrimoine) ont été choisis, permettant de constituer six groupes de quatre-cinq personnes.

6 L'équipe organisatrice avait conduit des pré-ateliers sur le terrain et prévenu les populations de la $\mathrm{CR}$ de nos enquêtes, en s'appuyant sur leurs points focaux, la plupart riziculteurs joola, médiateurs entre les « experts » et les « locaux ». La méthodologie, les données acquises et les attendus ont été présentés aux participants et les groupes constitués en s'efforçant de combiner des compétences et expertises complémentaires.

7 Les visites de terrain par groupes thématiques (Figures 2A et 2B: deux des groupes thématiques) ont donné lieu à la réalisation de cartes des terroirs ancien et actuel et de transects selon les thèmes ciblés, aux recueils plus ciblés d'informations à travers des entretiens individuels et collectifs (calendrier des activités, généalogie, ...) et à des séances de présentation et de restitution de nos travaux, tous ces outils visant à un coapprentissage de savoirs.

Figure 2 : Deux des groupes thématiques de l'atelier de Cabrousse

2A : Groupe de Diembéring 


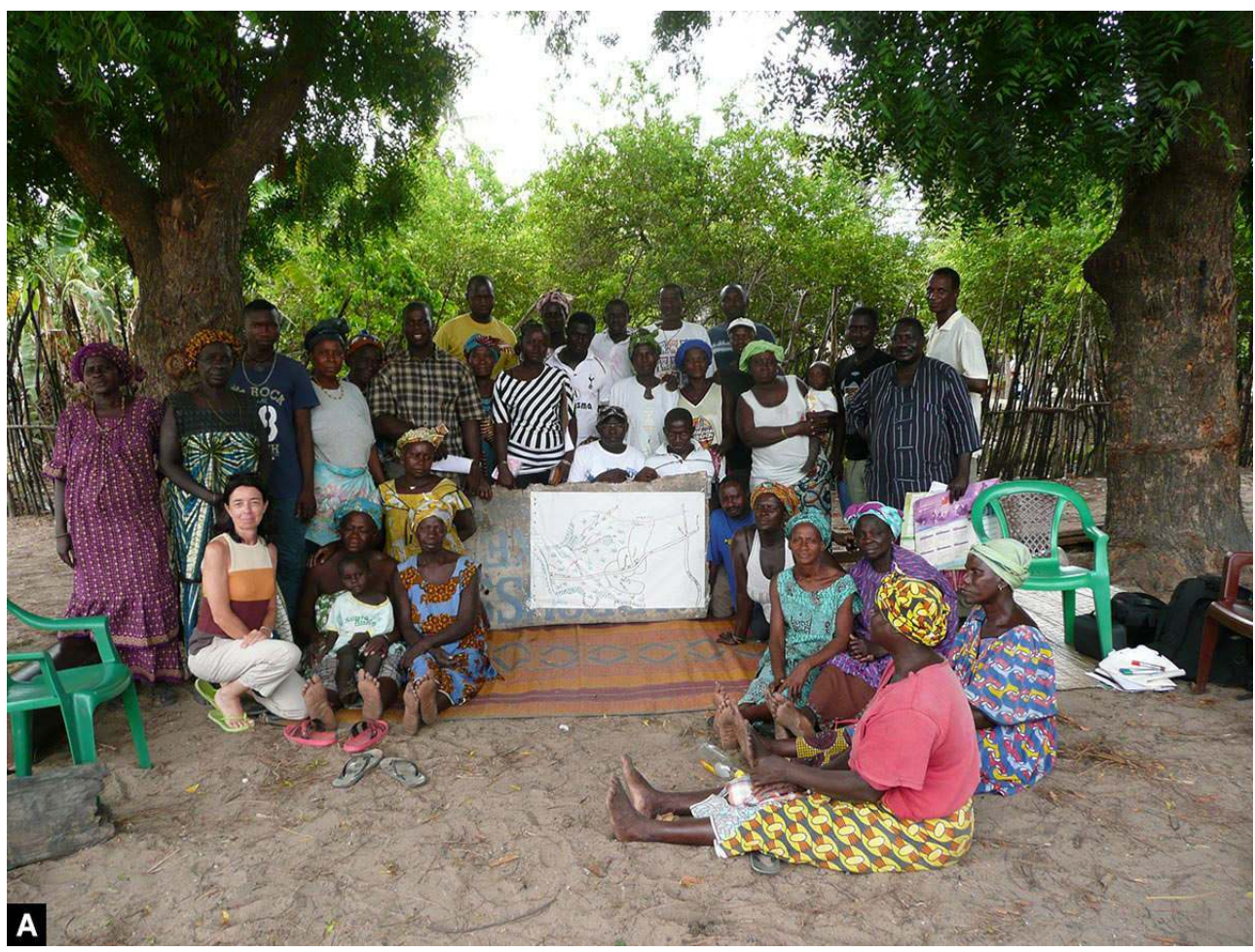

(c) M.C. Cormier-Salem

2B : Groupe de Bouyouye

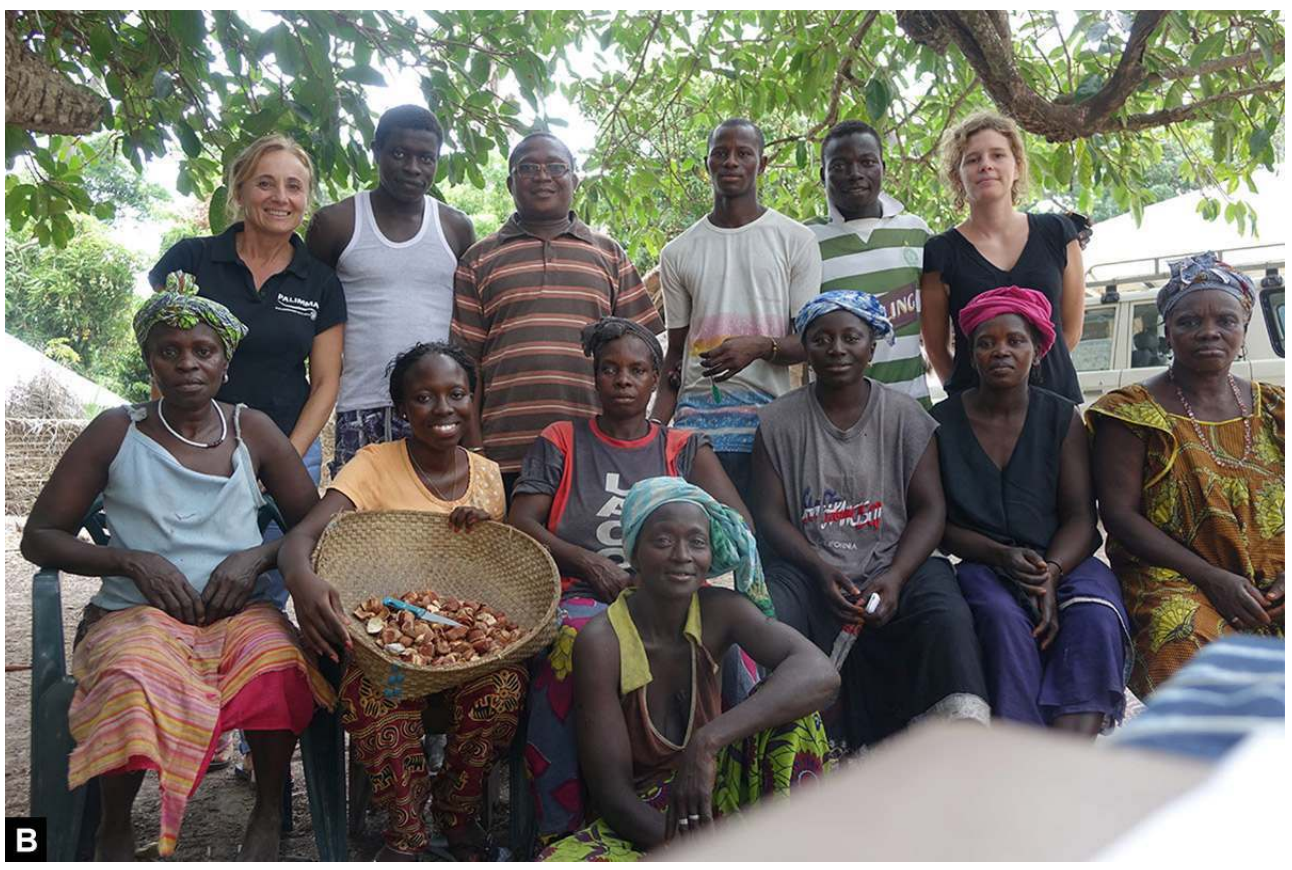

(C) F. Chlous

8 La troisième et dernière étape de la démarche méthodologique est la restitution, une phase très importante car permettant de valider, avec les populations locales, les données collectées sur le terrain. 


\section{Les principaux résultats}

9 Les résultats de l'expérience de cartographie participative menée sur le terrain de la Basse Casamance sont développés en détail dans les articles de Ehemba et al. (2017) et Sané et al. (2017). Nous nous contentons ici d'en faire la synthèse et d'en tirer les principales leçons. La commune de Diembéring (Figure 1), terrain d'application de cette expérience, est un site transfrontalier d'environ $430 \mathrm{~km}^{2}$ et $65 \mathrm{~km}$ de plage qui présente aujourd'hui de grands enjeux sur lesquels il fallait s'interroger en termes de recherchedéveloppement. Ces enjeux concernent les vulnérabilités littorales, à la fois environnementales (élévation du niveau de la mer, érosion côtière, irrégularités pluviométriques, salinisation des eaux et des sols, perte de biodiversité, etc.) et sociopolitiques (bétonisation du littoral avec le développement du tourisme, doublement de la population depuis 1968, mais aussi fort exode rural, se traduisant par un manque d'entretien des rizières, etc.). Ces profondes mutations exacerbent les compétitions pour le contrôle des ressources et des espaces, qui se traduisent par une insécurité foncière, de nouveaux usages liés aux projets d'exploitations minières (zircon, phosphate, etc.), le tout, dans un contexte d'instabilité politique (guerre civile depuis plus de 30 ans) et de fortes mobilités transfrontalières.

L'approche diachronique (sur un pas de temps de 50 ans), systémique (interaction des dynamiques écologiques et sociales) et inclusive (participation de tous les acteurs) a mobilisé divers outils, qui ont conduit à des produits, aux résultats tangibles. Si le « profil historique " a surtout été un outil d'échanges entre les différentes générations des communautés interrogées, les cartes des terroirs, anciens (environ 1950) et actuels (2014-15), des trois localités (Diembéring, Cabrousse et Bouyouye) ont permis de décliner les contenus et de révéler les différents usages des terroirs de communautés rurales (cf. Figure 3). Ehemba et al. (2017) considèrent la cartographie comme l'une des clés de lecture de la question foncière car, mise en œuvre de manière participative et fonctionnelle, elle permet à des populations de s'approprier d'une nouvelle manière leur territoire et de développer de manière concertée des outils de sécurisation du foncier. L'apport des transects, réalisés en salle puis complétés sur le terrain, à micro-échelle, a été déterminant dans la vérification et la validation des connaissances identifiées par les populations locales elles-mêmes (cf. Figure 4). Les entretiens réalisés sur les deux thématiques retenues (riz et riziculture, patrimoine) ont, pour leur part, permis d'approfondir la place respective des activités menées dans les terroirs et la dynamique des socio-écosystèmes. La restitution, étape ultime de cet exercice, a été un moment très important consacré à la validation par les populations locales des données recueillies sur le terrain. Elle a été aussi un moment de débats sur l'avenir de ces terroirs confrontés aujourd'hui à des enjeux multiformes, surtout fonciers, de plus en plus exacerbés, et qui impactent directement les conditions de vie des populations et questionnent sur la préservation et la sauvegarde des fondements sociaux traditionnels (cultures, cultes, pratiques sociales, rapports à leur environnement, etc.) de ces communautés humaines. Sané et al. (2017) précisent par ailleurs que la restitution constitue en ce sens un formidable outil de discussions et d'échanges intergénérationnels et entre populations locales et experts. Elle a été faite à deux niveaux, d'abord avec les populations locales et ensuite entre les groupes d'experts et les points focaux ayant conduit les travaux dans les différentes localités. 
Figure 3 : carte du terroir de Diembéring, élaborée par les acteurs de la communauté locale (dans une démarche participative)

3A : Terroir ancien

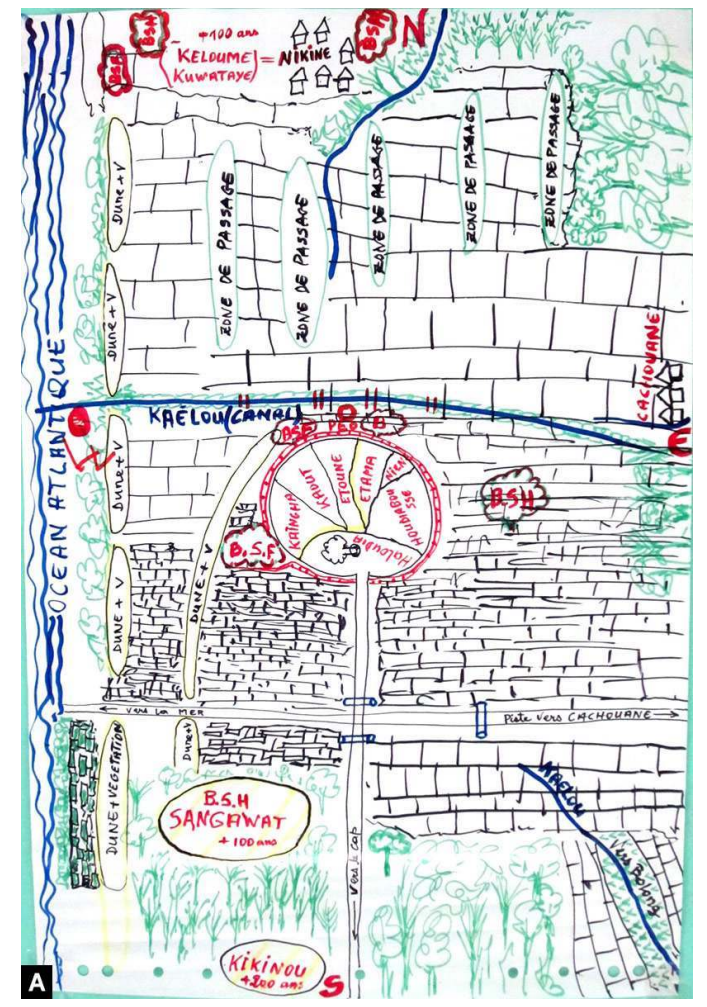

(c) T. Sané

3B : Terroir actuel 


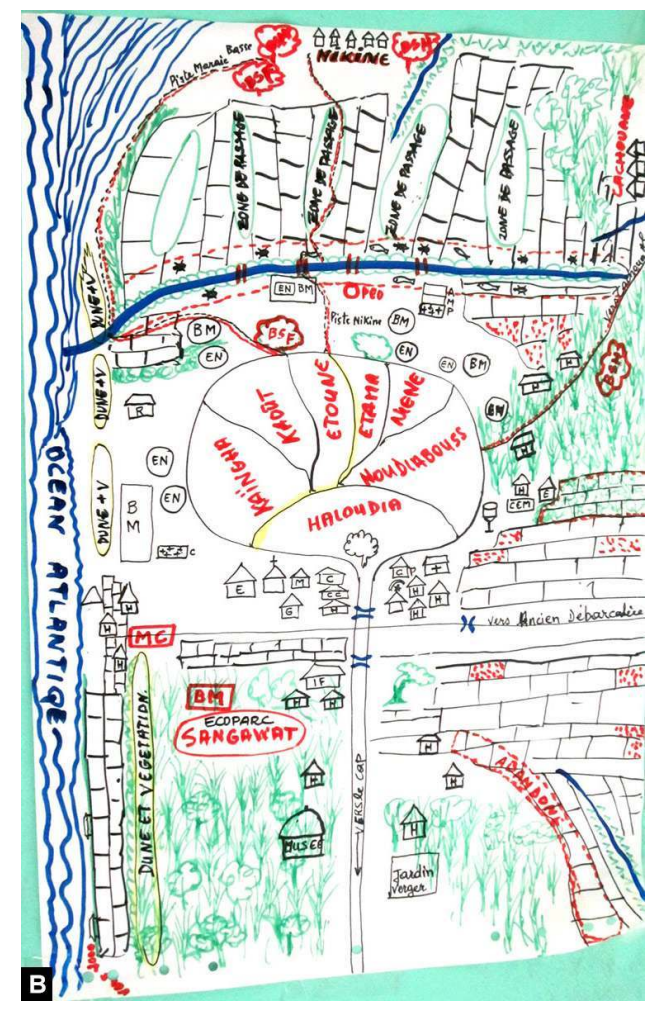

(c) T. Sané

Figure 4 : Transect spatial de Diembering

4A : Dessin participatif du transect de Diembéring

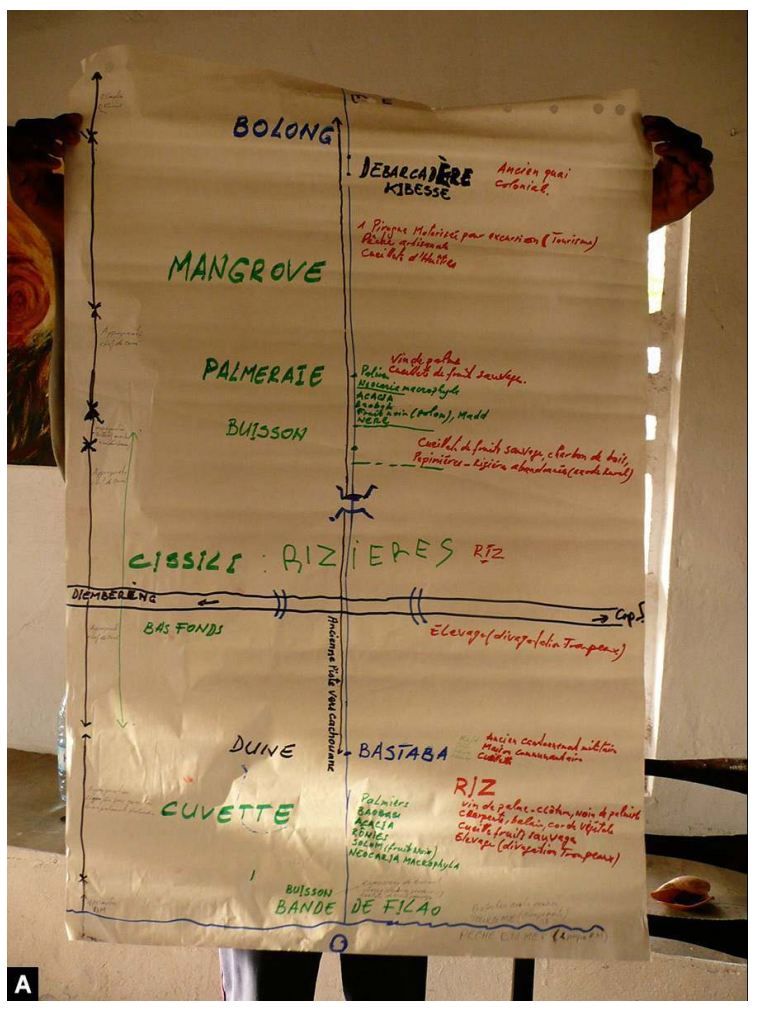

(c) M.C. Cormier-Sale

4B : Illustration de diverses unités de ce transect - La dune 


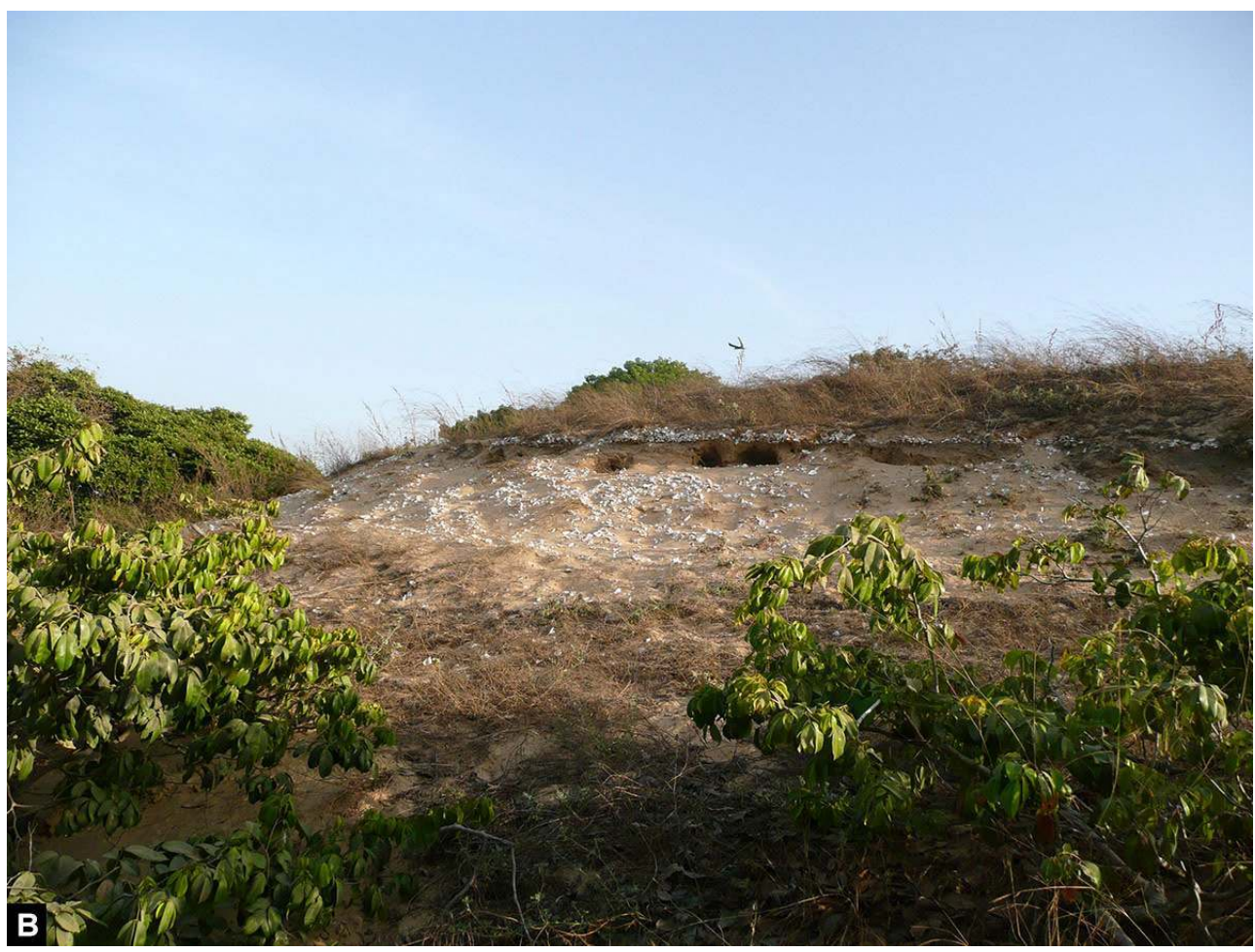

(c) M.C. Cormier-Salem

4C : Illustration de diverses unités de ce transect - Cuvette avec les rizières

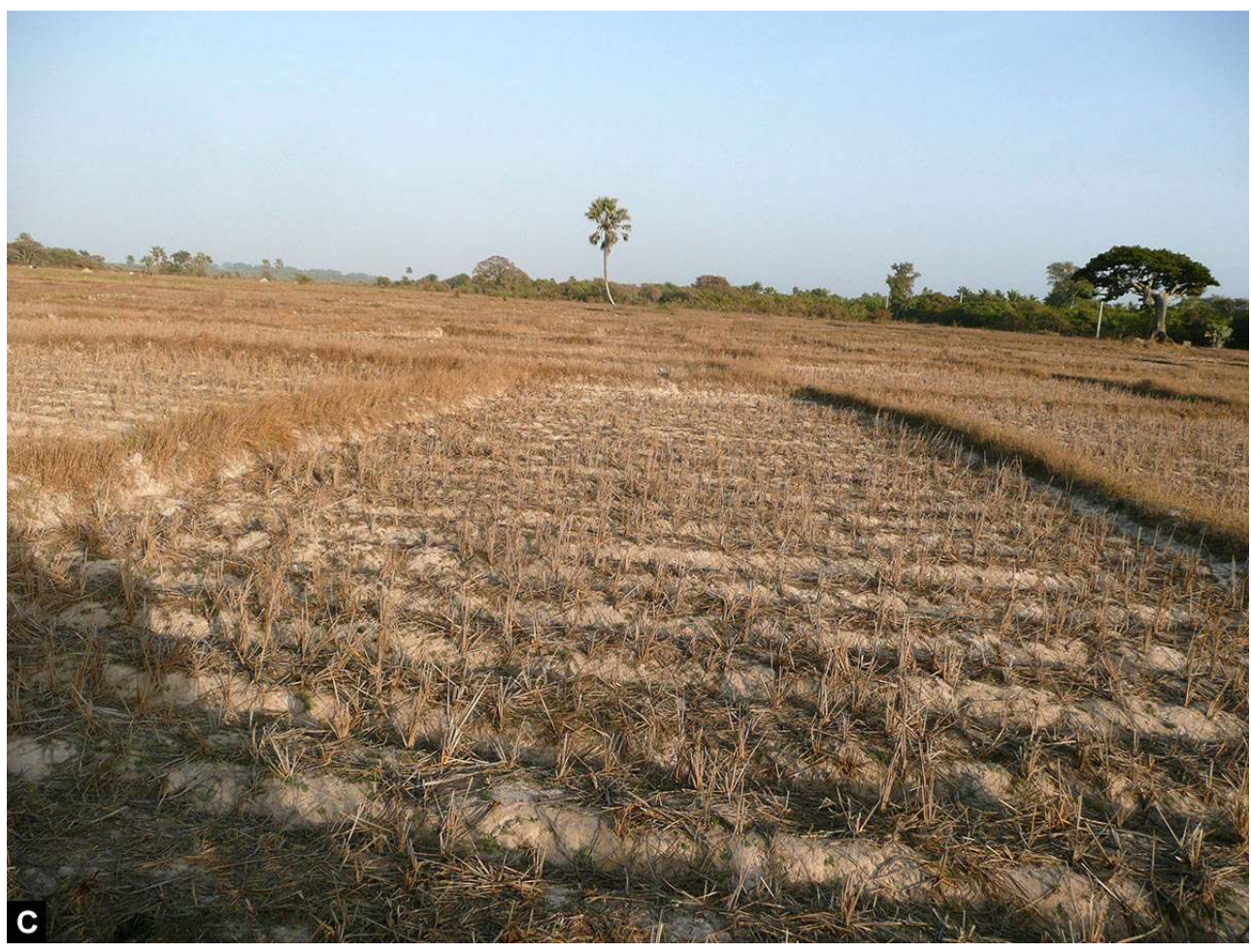

(c) M.C. Cormier-Salem

4D : Illustration de diverses unités de ce transect - La palmeraie 


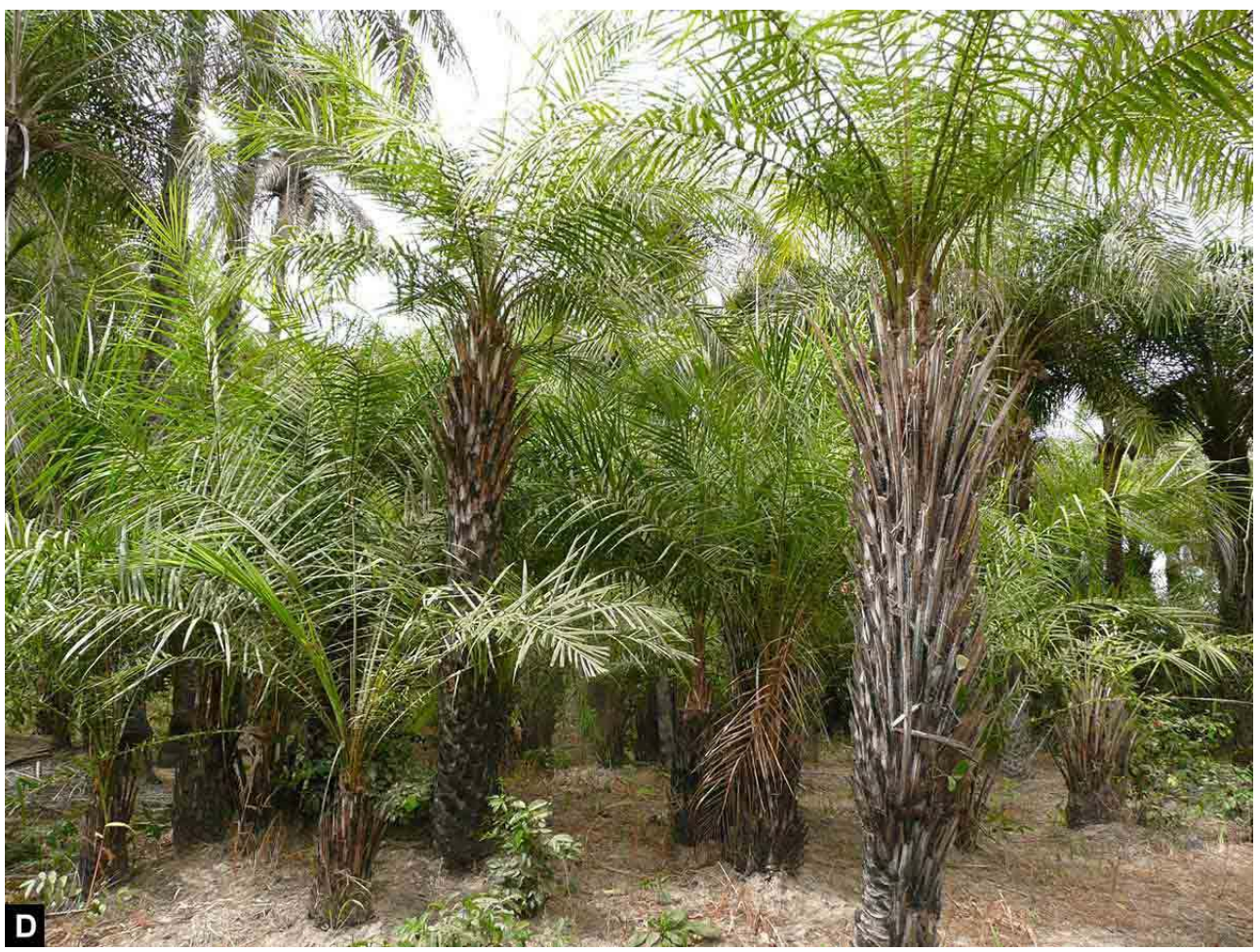

(c) M.C. Cormier-Salem

4E : Illustration de diverses unités de ce transect - La mangrove

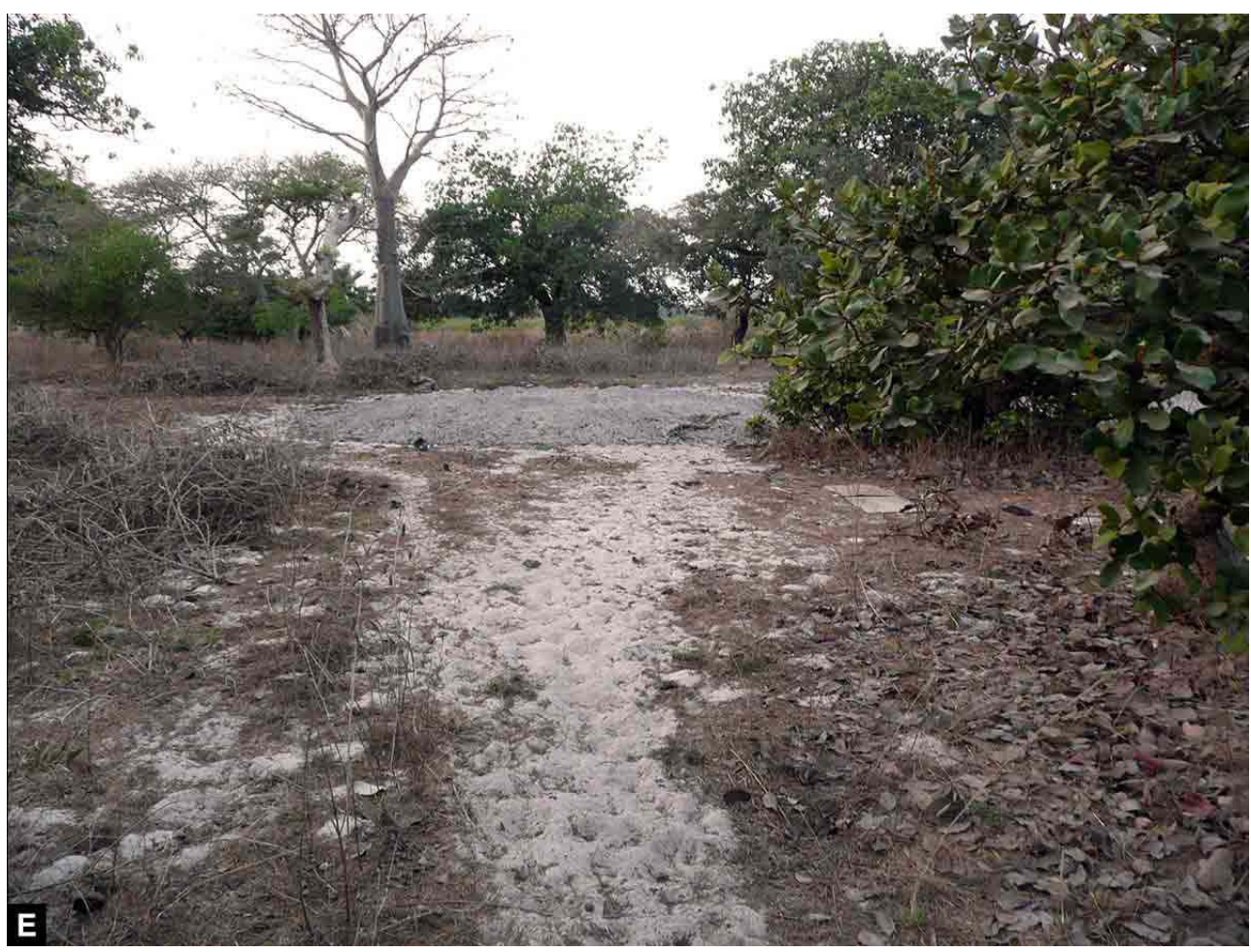

(c) M.C. Cormier-Salem

11 À l'issue de cet exercice, les populations locales ont manifesté leur satisfaction d'avoir participé et contribué activement à la production des connaissances sur leurs terroirs et exprimer le besoin de disposer des cartes et de toutes les données co-produites, ces 
éléments devant leur servir de base de réflexion et de plaidoyer pour l'aménagement de leurs terroirs respectifs. C'est dans cette perspective que des cartes ont été imprimées et mises à leur disposition. Les données collectées ont alimenté, en plus d'autres enquêtes menées dans les sites, la réalisation des monographies territoriales à travers les activités de la convention-programme AFD-GRDR. Dans le même sillage, elles ont également été mises à contribution dans l'élaboration du plan d'occupation et d'affection des sols (POAS) de la commune de Diembéring, une demande fortement exprimée par les autorités communales.

12 La valorisation scientifique de cet atelier de cartographie participative s'est traduite par de nombreuses conférences (Cormier-Salem et al. 2017, Sané et al. 2017, Ehemba et al. 2017), ainsi qu'un atlas en cours de publication (GRDR et al 2017). Ces publications, complémentaires, révèlent la complexité des problématiques et des enjeux qui touchent cette région littorale du Sénégal, surtout en termes de dynamiques. Elles mettent en exergue les rapports de pouvoirs entre différents villages autour des enjeux économiques (touristiques, miniers, agricoles, halieutiques, etc.) et fonciers au sein de cet espace.

\section{Discussion}

Lors de l'atelier et suite à nos travaux, plusieurs points ont fait l'objet de discussions, tels la finalité des cartes et leur durabilité, leur pouvoir et instrumentalisation, l'implication des acteurs et leur niveau de participation. Ici nous nous attachons à analyser les interactions complexes entre acteurs et les reconfigurations institutionnelles suscitées par ces démarches. En nous inspirant de la vaste littérature qui questionne, à travers les usages de la cartographie, la participation citoyenne (Péluso 1995, Amelot 2013, CormierSalem et al. 2017), il s'est agi d'identifier les diverses catégories d'acteurs et leur rôle respectif dans le processus,

Trois principales catégories d'acteurs se sont impliquées dans le processus de cartographie participative : les acteurs politiques, les habitants des villages et les experts. Les premiers, les acteurs politiques, sont omniprésents, à toutes les étapes du processus, mais en particulier à l'origine et à la fin, dans la mesure où ce sont eux qui promeuvent la démarche auprès des populations, qui les mobilisent et qui in fine ont le contrôle sur les produits issus du processus, décident et gouvernent. Dans la CR de Diembéring, ces acteurs sont représentés par des autorités déconcentrées et décentralisées, jouant un rôle clé dans le contexte actuel de la mise en œuvre de l'acte 3 de la décentralisation au Sénégal ${ }^{3}$. Néanmoins, les représentants du pouvoir traditionnel - chef du village choisi par les Sages et assisté par le conseil des Anciens; chef coutumier, gardien des autels des bois sacrés du terroir -, sont encore très respectés et ont une forte légitimité institutionnelle et religieuse. Intermédiaires entre les ancêtres et les boekin (fétiches), ce sont eux notamment qui règlent les conflits en première instance, qui définissent le calendrier des activités (aussi bien productives que rituelles comme le bukut, rite d'initiation et d'entrée dans les bois sacrés) et gouvernent les ressources et espaces collectifs du terroir, ou communs.

Les habitants des villages ont des fonctions et statuts différenciés, des "simples" informateurs aux facilitateurs et partenaires. Tous les habitants, hommes et femmes, aînés et cadets, locaux et nouveaux entrants, sont invités à participer au processus (Figure 5A). Néanmoins, dans la pratique, on constate de forts déséquilibres. En 
schématisant quelque peu, les participants aux enquêtes sont le plus souvent des hommes, âgés ou mariés et chefs de famille, instruits ou sachant parler français (la langue de la plupart des experts), voire écrire et dessiner une carte (selon les modes occidentaux ou cartésiens de représentation de l'espace). Sur ce dernier point, il est intéressant de constater la tendance actuelle à une prise de pouvoir de jeunes, de retour au village, qui sont passés par l'école et sont plus à l'aise pour répondre aux attentes des experts. Dans le village de Cabrousse, le poste de chef de village est désormais occupé par un jeune, ce qui manifeste la remise en cause du pouvoir coutumier des Anciens. Par ailleurs, il faut souligner la moindre participation des femmes, du moins en présence des hommes, mais aussi la faible sensibilisation des acteurs, ce qui s'explique sans doute par un temps de préparation trop court.

En revanche, les points focaux et les enquêteurs, issus des associations et communautés locales, ont joué un rôle fondamental. Dans chaque localité retenue (Diembéring, Bouyouye et Cabrousse), deux personnes ont été choisies comme points focaux c'est-àdire des personnes ressources et des facilitateurs pour toutes les démarches entreprises dans ces localités. Ils constituent l'interface entre les experts et la population locale. Ils jouent un rôle déterminant dans le travail du fait qu'ils ont été la porte d'entrée et ont mobilisé la population dans leurs localités respectives. Ils sont présents à toutes les étapes du processus, notamment lors de la restitution et du transfert des résultats aux habitants.

17 La troisième catégorie d'acteurs comprend les experts, ceux qui maîtrisent les outils conceptuels et les techniques (étudiants, enseignants, chercheurs en sciences sociales et en sciences de l'environnement; géomaticiens et cartographes), qui sont spécialistes, à des degrés divers, de l'élaboration des cartes et de l'analyse spatiale. Ils sont tout à la fois initiateurs, concepteurs, animateurs et opérateurs. Ils sont en effet à l'initiative de la démarche, du choix des outils, des sites d'étude. Ce sont eux qui ont élaboré les Termes De Référence (TDR) et les guides d'entretien, le cadre logique et la mise en oeuvre sur le terrain, en charge tout aussi bien de la logistique que de la constitution des équipes de travail pluridisciplinaires. Ils ont un rôle essentiel d'animation et de management des personnes avec le recrutement des points focaux, la formation des enquêteurs, l'encadrement des stagiaires, mais aussi la convocation et l'animation des réunions. Le succès de la participation dépend largement de leur capacité à impliquer les personnes ressources aux diverses étapes $\mathrm{du}$ processus (information, communication, sensibilisation, consultation, concertation, négociation, et co-construction) et de s'assurer de la représentation de toutes les parties-prenantes à la démarche, depuis l'amont (conception du projet, production des données) jusqu'à l'aval (prise de décision, contrôle et restitution de l'information). Le simple énoncé de ces tâches révèle les défis de cette démarche qui, plus est, se veut interdisciplinaire, collective et inclusive. Or point n'est besoin de s'appesantir sur le fait qu'au sein même des experts, les différences de statuts et de rôles sont évidentes en fonction de leur origine, formation, implication dans le processus, etc., mais aussi en fonction de leurs objectifs, attendus et motivations.

Les trois catégories d'acteurs sont traversées de conflits d'intérêts, de jeux de pouvoirs et de savoirs et interagissent entre elles dans une dialectique complexe de complicité, complémentarité et reconnaissance réciproque mais aussi manipulation et instrumentalisation (Gould \& Bailly 1995, Amelot 2013).

19 L'atelier de cartographie participative de Cabrousse se voulait être un exercice de formation interdisciplinaire et interculturelle entre des acteurs aux statuts fort divers avec schématiquement trois équipes selon l'origine (locale, PALOC, PATEO), trois types 
d'expertise ou de connaissances (techniques infographiques, scientifiques, savoirs locaux) (Figures 5). Mais, pour certains experts, il s'agissait aussi de tester une démarche inductive (Bourdieu et al. 1968), de produire des cartes, sans aucune demande locale, sans même un cadrage préliminaire (selon la posture de «laisser dire et laisser faire ») avec néanmoins une portée forte en terme d'aménagement du territoire et donc une visée très appliquée, dénotant une certaine dérive entre les objectifs initiaux et la valorisation finale. On est ainsi en droit de s'interroger sur l'utilisation, voire la manipulation des cartes, sans que tous les acteurs en maîtrisent les usages.

Figure 5 : Catégories d'acteurs participants

$5 \mathrm{~A}$ : Les populations locales

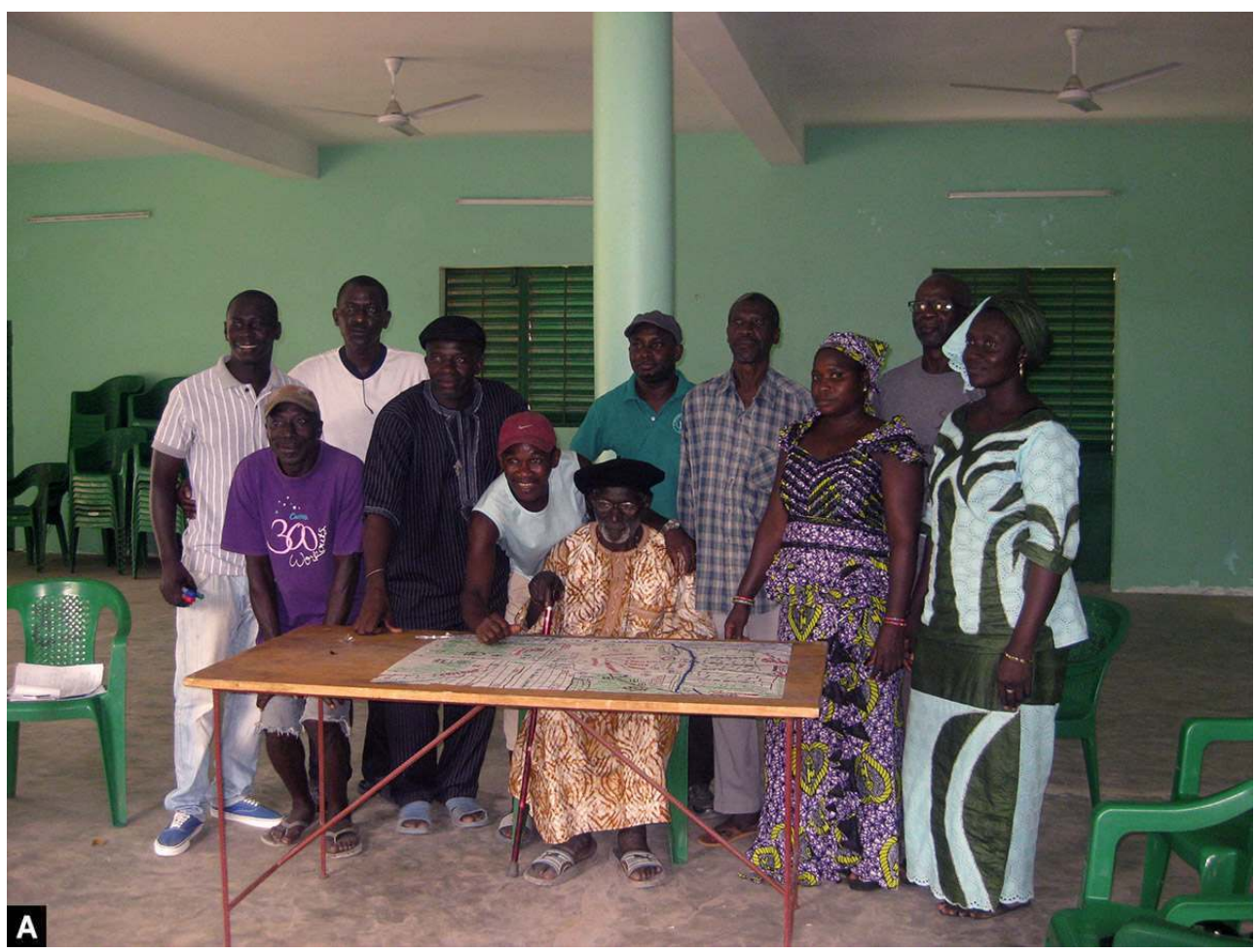

(C) M.C. Cormier-Salem

5B : Les jeunes et les experts techniciens 


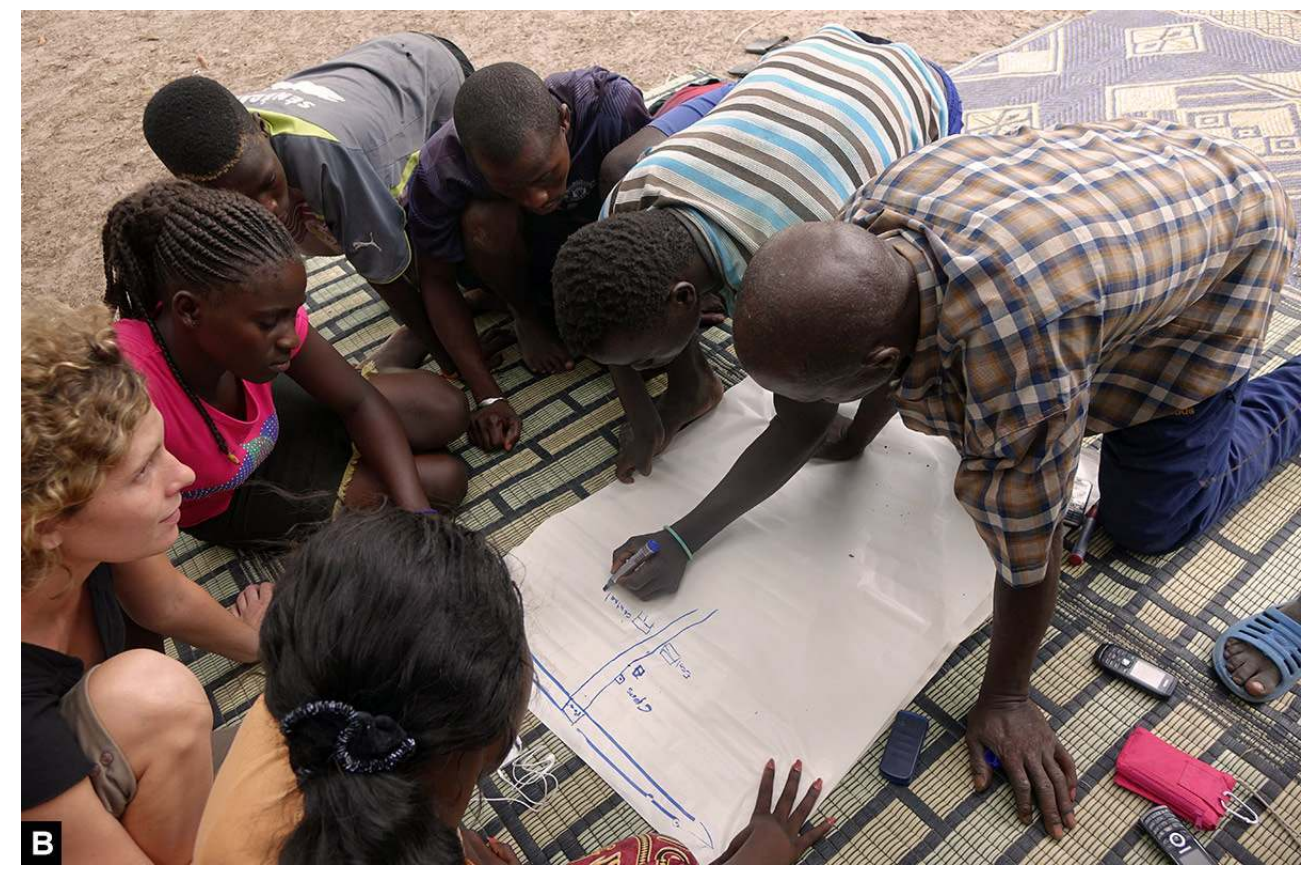

(c) F. Chlous

$5 \mathrm{C}$ : Les experts et points focaux

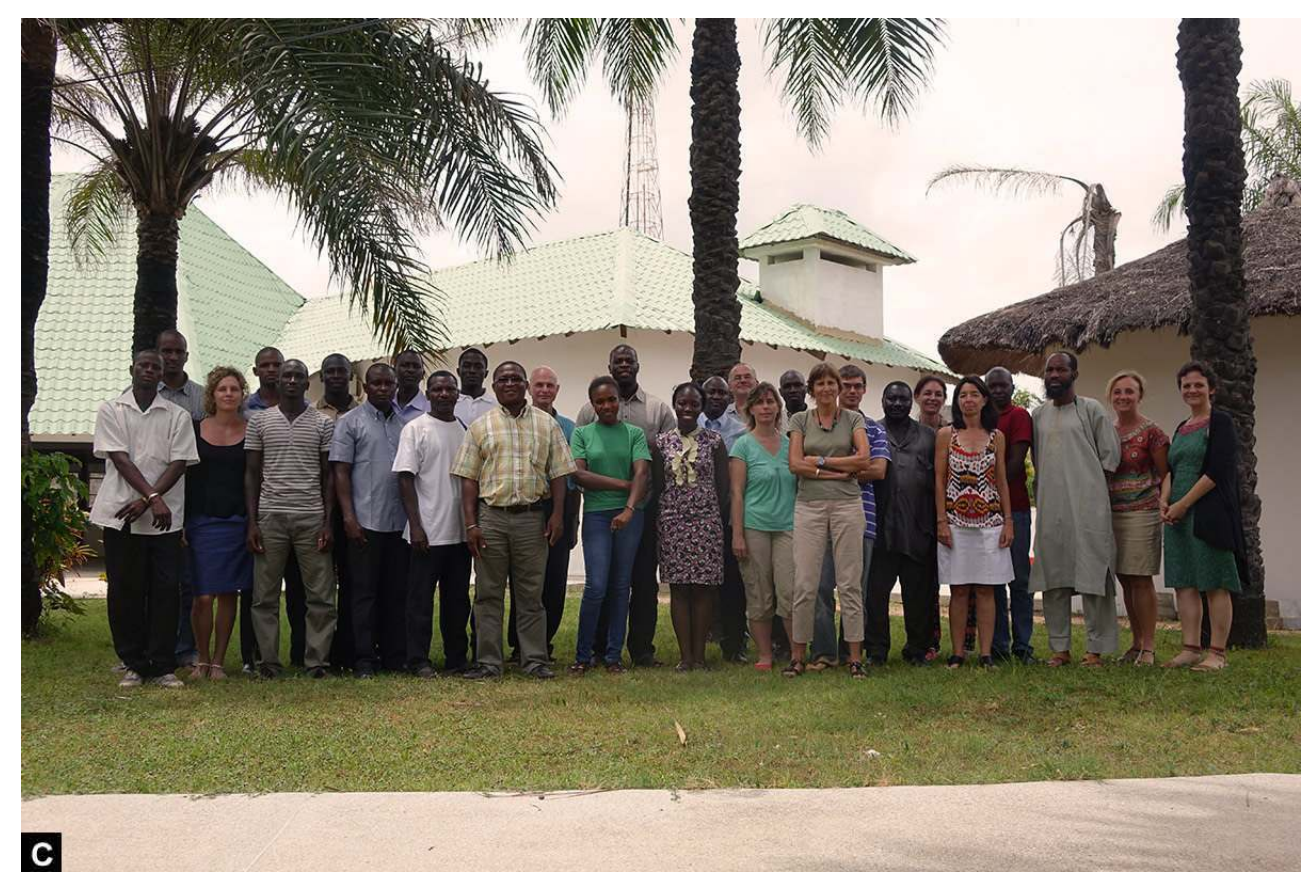

(C) M.C. Cormier-Salem

20 Nonobstant ces interrogations sur l'illusion de la connaissance partagée ou les limites de la trans-appropriation des savoirs, les suites de cet atelier (Ehemba et al. 2017) ont montré l'importance des cartes communautaires comme plaidoyer pour la CR et le rôle du POAS (Plan d'Occupation et d'Affectation des Sols) comme outil de médiation et de prospective autour d'un projet de territoire partagé. Ainsi loin d'être un simple exercice académique ou pédagogique, cet atelier a débouché sur des propositions d'aménagement concertées du territoire et de résolution (ou de débats contradictoires/ polémiques) des conflits fonciers. 
21 De fait, l'exercice a permis de : dresser un portrait du village dans sa globalité sur les deux thèmes choisis, patrimoine et riz et riziculture; avoir une compréhension globale des dynamiques et enjeux des terroirs rizicoles (opportunités/contraintes); tirer des leçons sur le cadre méthodologique mis en œuvre; avoir des éléments de base permettant d'élaborer un diagnostic territorial.

\section{Conclusion}

Issue d'une démarche collective, initiée et encadrée par des experts, limitée dans le temps (une semaine) et l'espace (la communauté rurale de Diembéring), l'expérience de cartographie participative mise en œuvre en Casamance n'échappe pas aux critiques mises en avant dans d'autres contextes. De nombreux travaux soulignent ainsi que la cartographie participative n'est pas neutre, mais suscite ou révèle des conflits, oppositions et résistances (Gould \& Bailly 1995, Péluso 1995, Reyes-García 2012, Amelot 2013); cette technique peut être utilisée pour défendre une cause, soutenir les revendications des citoyens et les mobiliser (Liagre \& Nonjon 2012, Bryan 2015). La réplicabilité de la méthodologie de terrain est délicate et il faut souligner les fréquentes tensions entre montée en généralité ou généricités et spécificités: les informations récoltées, fines, concrètes, sont partielles, voire partiales, ponctuelles et fragmentaires (Joliveau 2004).

23 L'exercice néanmoins est riche et invite à explorer trois pistes de réflexion :

- la première tient à la carte et à son usage. Pour tous les participants à l'atelier, il est apparu que le processus inter-acteurs l'emportait sur le produit stricto sensu. La démarche, impliquant diverses catégories d'acteurs (politiques, locaux, experts « étrangers ») est innovante car elle repose sur de nouvelles modalités d'acquisition des connaissances; elle contribue à la création d'un réseau d'acteurs et débouche sur de nouveaux espaces de dialogue, qui sont autant d'outils de mobilisation collective et de contre-pouvoir.

- La deuxième piste concerne l'alibi du participatif de la cartographie et interroge sur les risques de dilution des frontières entre sciences, sociétés et politique, sur les tensions entre participation universelle et participation limitée aux experts et les dérives politiques et éthiques inhérentes à ces démarches (Cormier-Salem et al. 2017). Un regard extérieur, plus neutre que celui des enquêteurs et points focaux issus du village, peut s'avérer pertinent. Un équilibre est à trouver entre les besoins explicités par les habitants et l'expertise des intervenants extérieurs, sans parler de la représentativité des communautés locales, et du rôle de l'État, aux gouvernementalités différenciées.

- la troisième piste porte sur l'engagement des chercheurs qui mobilisent ces démarches de participation, sur leurs biais scientifiques, tels les changements d'échelles spatiales et temporelles ou encore sur le statut des connaissances produites (leur production, circulation et application).

24 Ces pistes invitent à une claire posture, pour une géographie militante ou pour une approche de géographie engagée, critique, sinon radicale (Death 2014, Chartier \& Rodary 2016). 


\section{BIBLIOGRAPHIE}

Amelot X. 2013 - Cartographie participative pour le développement local et la gestion de l'environnement à Madagascar : Empowerment, impérialisme numérique ou illusion participative ? L'information géographique 77 (4) : 47-67.

Arnstein S.R. 1969 - A Ladder of Citizen Participation. American Institute of Planners Journal 35 (4) : 216-224.

Bourdieu P., Chamboredon J.C. \& Passeron J.C. 1968 - Le métier de sociologue. Préalables épistémologiques. Paris, Mouton, Bordas, $430 \mathrm{p}$.

Bryan J. 2015 - Participatory mapping. In : T. Perreault, G. Bridge \& J. McCarthy, The Routledge handbook of political ecology. Abingdon, Oxon ; New York, NY, Routledge : 249-262.

Burini F. 2005 - La cartographie participative dans la recherche de terrain en Afrique : le cas des villages riverains au Parc Régional W. Policy Matters, special edition WRegional Park, IUCN.

Burini F. 2008 - La cartographie participative et la pratique du terrain dans la coopération environnementale : la restitution des savoirs traditionnels des villages de l'Afrique subsaharienne. À travers l'espace de la méthode : les dimensions du terrain en géographie, Arras, France.

Chambers R. 1991 - Rapid and Participatory Rural Appraisal. Brighton (R.-U.), Institute of Development Studies.

Chambers R. 2006 - Participatory Mapping and Geographic Information Systems: Whose Map? Who is Empowered and Who Disempowered? Who Gains and Who Loses? EJISDC 25 (2) : 1-11.

Chartier D. \& Rodary E. 2016 - Manifeste pour une géographie environnementale. Géographie, écologie, politique. Paris, Sciences po. Les presses, Domaine Développement durable, $412 \mathrm{p}$.

Cormier-Salem M-C., Descroix L. \& Diakhaté M. (Ed.) 2017 - Sciences participatives et gouvernance des patrimoines et territoires des deltas. Actes du colloque international de Saint Louis (11-14 mai 2016). L'Harmattan, Dakar, 373 p.

Death C. 2014 - Critical Environmental Politics. New-York, Routledge, 350 p.

Ehemba F., Le Coq Y., Ruë O., Niokane M., Bodivit M., Seck P., Diallo M., Agossou S., Descroix L., Sané T. \& Fabre M. 2017 - Une cartographie participative pour bâtir une connaissance concertée des mutations de territoire dans l'espace frontalier sénégalo-bissao-guinéen. In : M.C. CormierSalem, M.M. Diakhaté \& L. Descroix (Ed.) Sciences participatives et gouvernance des patrimoines et territoires des deltas. Dakar, Karthala, Actes du colloque PATEO/ PRCM : 167-182.

Gould P. \& Bailly A. (Ed.) 1995 - Le pouvoir des cartes. Brian Harley et la cartographie. Paris, Anthropos, 120 p. (Collection Géographie).

GRDR-IRD-UASZ 2017 - Des territoires littoraux en mouvement. Diversité, dynamiques et mutations du sud-ouest du Sénégal et du nord-ouest de la Guinée-Bissau (Oï, Cacheu, Ziguinchor, Sédhiou). Atlas GRRIRD-UASZ/ convention programme AFD (impression en juin 2017)

Gueye B. \& Schoonmaker-Freudenberger K. 1991 - Introduction à la méthode accélérée de recherche participative [MARP] : rapid rural appraisal, quelques notes pour appuyer une formation pratique.

Londres, International Institute for Environment and Development. 
Habert E. 2014 - Cartographie participative : méthodes et outils. Communication Atelier de cartographie participative. LMI PATEO-Université de Ziguinchor-GRDR, Cabrousse (Casamance, Sénégal), 30 juin-4 juillet 2014 .

Joliveau T. 2004 - Géomatique et gestion environnementale du territoire. Recherches sur un usage géographique des SIG. Mémoire d'habilitation à diriger des recherches. Rouen, Université de Rouen, 615 p.

Lavigne Delville P. 2011 - Du nouveau dans la « participation »? : populisme bureaucratique, participation cachée et impératif délibératif. In : Jul-Larsen E., Laurent P.-J., Le Meur P.-Y. \& Léonard E. (Ed.) Une anthropologie entre pouvoirs et histoire. Conversations autour de l'œuvre de JeanPierre Chauveau. Paris/Marseille/Uppsala, Karthala-IRD-APAD :160-187.

Liagre R. \& Nonjon M. 2012 - Une cartographie participative est-elle possible ? EspacesTemps.net, Textuel, http://espacestemps.net/document9495.html

Palsky G. 2010 - Cartes participatives, cartes collaboratives. La cartographie comme maïeutique. CFC $25: 49-59$

Péluso N.L. 1995 - Whose woods are these? Counter-mapping forest territories in Kalimantan, Indonesia. Antipode 27 (4) : 383-406.

Reyes-García, V., Orta-Martnez M., Gueze M., Luz A.C., Paneque-Glvez J., Maca M.J., Pino J. \& Teame T.B.S. 2012 - Does Participatory Mapping Increase Conflicts? A Randomized Evaluation in the Bolivian Amazon. Applied Geography 34 : 650-658.

Sané T., Cormier-Salem M.C., Dièye E.H. Balla, Descroix L., Fabre M., Habert E., Ehemba F., Bodivit M., Demba Ba B. \& Mendy V. 2017 - La cartographie participative comme outil d'aide à la compréhension des dynamiques territoriales : application sur un terrain de Basse Casamance (Sénégal). In : M.C. Cormier-Salem, M.M. Diakhaté \& L. Descroix (Ed.) Sciences participatives et gouvernance des patrimoines et territoires des deltas. Dakar, L'Harmattan, Actes du colloque PATEO/ PRCM : 265-290.

\section{NOTES}

1. Cette école thématique a été organisée grâce aux soutiens financiers de l'UMR PALOC «Patrimoines Locaux et Gouvernance », du LMI PATEO «Patrimoines et territoires de l'eau » et d'une «Action Incitative » de l'IRD. Le comité d'organisation était composé de l'ONG Groupe de Recherches et de Réalisation pour le Développement Rural/GRDR, pôle littoral Sud et de l'UASZ (Université Assane Seck de Ziguinchor) appuyé par l'équipe de direction de PATEO et de PALOC.

2. Sont venus de l'UMR PALOC de France six chercheurs de sciences sociales: F. Chlous (anthropologue) et Y. Girault (muséologue) du MNHN; D. Guillaud (anthropogéographe), P. de Robert (anthropologue), S. Duvail (géographe), L. Descroix (hydrologue), de l'IRD et E. Habert du Service de cartographie de l'IRD. Du Sénégal, ont participé des collègues et étudiants des universités partenaires (Université Gaston Berger/UGB de Saint Louis, Université Cheikh Anta Diop/UCAD de Dakar et Université Assane Seck de Ziguinchor/UASZ), de l'ONG GRDR, (Yvan Lecoq, Melig Bolivit) et de l'IRD (M.C. Cormier Salem, géographe et M. Fabre, géomaticienne)

3. Depuis 2013 , le Sénégal a engagé la $3^{\text {ème }}$ phase de la réforme de sa politique de décentralisation qui s'est traduite par la "communalisation intégrale» (les anciennes communes rurales ont désormais les mêmes compétences que les communes urbaines), l'érection des départements en collectivités locales et la suppression des régions en tant que collectivités locales. 


\section{RÉSUMÉS}

L'objectif de cet article est de montrer la dynamique collective multi-partenariale insufflée par un atelier de cartographie participative, organisé dans la commune rurale de Diembéring en Casamance au sud-ouest du Sénégal, en juillet 2014. Il s'agissait de saisir l'organisation, passée et actuelle, des espaces villageois et leurs usages à partir des cartes de terroirs, réalisées par et avec les populations locales de trois localités de cette communauté rurale, Diembéring, Bouyouye et Cabrousse.

Cet atelier a permis de faire émerger les savoirs et savoir-faire traditionnels des communautés villageoises en termes de représentation de leurs espaces, mais aussi de mettre en lumière des questions souvent difficiles à appréhender, comme les conflits fonciers. Couplée avec d'autres outils d'enquêtes de terrain (entretiens, transects, profils historiques, calendrier agricole, etc.), la cartographie participative a mis en évidence la crise que traverse la riziculture en Casamance, et ses conséquences sur les fondements des communautés joola.

Au-delà de la production de cartes et de nouvelles connaissances co-construites, cet atelier a conduit à formuler explicitement les besoins des acteurs locaux et leurs questions sur l'avenir de leurs terroirs et à les accompagner dans leurs plaidoyers (engagement de dialogue multidimensionnel) auprès des autorités locales ou déconcentrées de l'État sénégalais. Il interroge par ailleurs les experts, scientifiques et techniques, sur leur posture intellectuelle et leur engagement dans la recherche-action.

The aim of this article is to show the collective multi-partnership dynamics, inspired by the participatory mapping workshop organized in the rural community of Diembering in Casamance, in southwest Senegal, in July 2014. The purpose of this workshop was to understand the past and present organization of the village's spaces and the uses of maps of the "terroirs", or communal territories, made by and with the local people of three localities of this rural community: Diembéring, Bouyouye and Cabrousse.

This workshop brought out the traditional knowledge and know-how of the local people in terms of representation of their spaces, but it also highlighted issues that are often difficult to apprehend, such as tenure conflicts. Coupled with other tools of field surveys (interviews, transects, historical profiles, agricultural calendar, etc.), participatory mapping pointed out the rice farming crisis in Casamance and its profound socio-cutural consequences on the Joola communities.

Beyond the production of maps and new co-constructed knowledge, this workshop led to explicitly formulate the needs of the local people and their questions about the future of their terroirs and to accompany them in their advocacy (commitment of multidimensional dialogue) with the local or deconcentrated authorities of the Senegalese State. It also was the occasion to ask scientific and technical experts about their intellectual posture, and their commitment to applied research. 
INDEX

Keywords : partipatory mapping, methodological framework, partnership, knowledge, spatial dynamics, Casamance, Senegal

Index géographique : Casamance, Sénégal

Mots-clés : cartographie, cadre méthodologique, partenariat, participation, engagement, dynamique territoriale

\section{AUTEURS}

\section{MARIE-CHRISTINE CORMIER-SALEM}

Géographe, Directrice de Recherche IRD, co-directrice du Laboratoire Mixte International Patrimoines et Territoires de l'Eau, UMR 208, PALOC, IRD/MNHN, Sorbonne Universités, 57 rue Cuvier, 75231 Paris cedex 05, France

marie.cormier@ird.fr

\section{TIDIANE SANÉ}

Géographe, Enseignant au Département de Géographie, point focal du Laboratoire Mixte International Patrimoines et Territoires de l'Eau, UFR Sciences et Technologies, Université Assane Seck de Ziguinchor (UASZ), BP 523 Ziguinchor, Sénégal tsane@univ-zig.sn 